\title{
ORIGINAL
}

\section{MEDIDAS DE EXPOSICIÓN A LA MOVILIDAD EN LAS LESIONES GRAVES POR TRÁFICO EN LA COMUNIDAD DE MADRID}

\author{
Luis Velázquez Buendía, María Felicitas Domínguez-Berjón, María D Esteban-Vasallo, Ricard Gènova \\ Maleras y Ana Clara Zoni.
}

Subdirección de Promoción de Salud y Prevención. Consejería de Sanidad. Comunidad de Madrid. Madrid. España.

\section{RESUMEN}

Fundamentos: La búsqueda de indicadores apropiados para estimar el riesgo de lesiones por tráfico es actualmente un área de interés relevante. El objetivo de este estudio fue realizar una descripción de la morbilidad hospitalaria y la mortalidad por lesiones por tráfico en la Comunidad de Madrid, según edad y sexo, utilizando y comparando entre sí tasas por población, por personas-km y por personas-horas.

Métodos: Estudio descriptivo transversal en la Comunidad de Madrid referido al período 2003-2005. Se estimaron y compararon por edad y sexo tasas de morbilidad y mortalidad por población, por personas-km recorridos y por personas-horas de desplazamiento. Se utilizaron como fuentes de información el Conjunto Mínimo Básico de Datos Hospitalarios de 2003 2005, la Encuesta Domiciliaria de Movilidad de 2004 en la Comunidad de Madrid y el registro de mortalidad del Instituto de Estadística de la Comunidad de Madrid.

Resultados: Se identificaron 7.413 altas hospitalarias y 1.046 defunciones. Las tasas poblacionales de morbilidad hospitalaria y mortalidad fueron de 62,24 y $9,20 / 100.000$ habitantes respectivamente en hombres, y de 23,80 y $2,97 / 100.000$ en mujeres, siendo las más elevadas en varones de 16-24 años (119,27 altas y 12,00 fallecidos por 100.000 habitantes). Las tasas por exposición más altas correspondieron a mujeres de 65 y más años: 649,78 altas y 96,72 fallecidas por $10^{9} \mathrm{~km}$, y 13,11 altas y 1,95 fallecidas por 10 horas de desplazamiento.

Conclusiones: La morbi-mortalidad fue mayor en hombres en todos los indicadores. Los indicadores basados en la exposición a la movilidad, frente a las tasas poblacionales, reducen la morbi-mortalidad de lesiones por tráfico en hombres y en edades jóvenes y aumentan la misma en edades avanzadas.

Palabras clave: Accidentes de tránsito. Medición de Riesgo. Medidas. Mortalidad. Morbilidad. Lesiones.

Correspondencia

Luis Velázquez

C/ San Martín de Porres, 6, $1^{\text {a }}$ planta.

Servicio de Informes de Salud y Estudios

28035 Madrid.

luis.velazquez@salud.madrid.org

\section{ABSTRACT Mobility Exposure Measures in Serious Road Traffic Injuries in Madrid, Spain}

Background: The search of suitable indicators for estimating the risk of road traffic injuries is nowadays a relevant topic. The objective of this study was to carry out a comparative description of mortality and inhospital morbidity by age and sex, using population rates and mobility exposure related indicators.

Methods: Cross sectional study in the Community of Madrid, 20032005. Population rates and mortality and morbidity rates per billion of persons-kilometers travelled and per million of persons-hours travelled were estimated and compared by age and sex. The Minimum Basic Hospital Discharge Data Set, the 2004 Mobility House Survey of the Community of Madrid and the mortality register of the Statistic Institute of the Community of Madrid were used as information sources

Results: 7,413 hospital discharges and 1,046 deaths were identified. Morbidity and mortality population rates in men were 62.24 and 9.20 respectively, and in women 23.80 and 2.97 per 100,000 inhabitants, being the highest rates those for men aged 16-24 years (119.27 hospital discharges and 12.00 deaths per 100,000 inhabitants). Women of 65 years and older showed the highest mobility related rates: 649.78 hospital discharges and 96.72 deaths per $10^{9} \mathrm{~km}$, and 13.11 hospital discharges and 1.95 deaths per $10^{6}$ travelled hours.

Conclusions: Morbidity and mortality were higher in men for the three indicators. Rates referred to mobility exposure, faced to population rates, decrease mortality and morbidity due to road traffic injuries in men and young ages and increase both in advanced ages.

Keywords: Traffic Accidents. Risk Assessment. Measures. Mortality. Morbidity. Injuries.

DOI: 


\section{INTRODUCCIÓN}

El análisis comparativo de las lesiones por tráfico (LT) requiere indicadores adecuados que incluyan la movilidad de las personas. Las tasas poblacionales, como medida epidemiológica tradicional, son adecuadas para comparar el impacto de las LT en la salud de una población con el impacto causado por otras enfermedades, pero cuando se trata de comparaciones de las LT entre sí (por edades, regiones, tipos de vehículo, etcétera) o de evaluar programas preventivos, son necesarios indicadores más precisos en la estimación del riesgo que controlen la exposición, siendo los denominadores más utilizados los vehículos-km, personas-km y personas-horas de desplazamiento ${ }^{1-4}$. El primero de estos es fácil de obtener a través de las estadísticas oficiales de tráfico, pero refleja la movilidad de los vehículos y no la de las personas. Los otros dos requieren la realización de encuestas poblacionales de movilidad no siempre disponibles. En España se han publicado hasta la fecha dos estudios que utilizan como medida de exposición las personas-horas ${ }^{5,6}$.

La fuente de datos sobre morbi-mortalidad más habitual en los estudios de LT es la fuente policial. Tiene el inconveniente de producir una infra-notificación de casos $^{7-9}$ y no aportar información precisa sobre el tipo de lesión y gravedad de la misma. La utilización de fuentes sanitarias para complementar la fuente policial es una práctica habitual ${ }^{7}$. En España, el Conjunto Mínimo Básico de Datos de Alta Hospitalaria (CMBDAH) registra las altas hospitalarias de todos los hospitales públicos y en algunas regiones, como la Comunidad de Madrid (CM), también de todos los privados. Aunque se ha destacado la utilidad del CMBDAH como fuente de información para el estudio de $\mathrm{LT}^{8,10} \mathrm{su}$ explotación con este fin no es frecuente.

El objetivo de este estudio fue realizar una descripción de la morbilidad hospitalaria y la mortalidad por lesiones por tráfico en la Comunidad de Madrid, según edad y sexo, utilizando y comparando entre sí tasas por población, por personas-km y por personas-horas.

\section{MATERIAL Y MÉTODO}

Diseño del estudio. Se realizó un estudio descriptivo transversal sobre las altas hospitalarias y fallecimientos por LT en residentes en la CM durante los años 2003-2005.

Fuentes y definiciones. Las definiciones de alta hospitalaria y fallecimiento por LT se basaron, con ligeras modificaciones, en las establecidas en 2007 por el Grupo de trabajo de la Sociedad Española de Epidemiología sobre medida del impacto en salud de las lesiones por tráfico en España ${ }^{11}$. No se incluyeron como altas hospitalarias por LT los casos en los que figuraba como régimen de financiación "Aseguradora de accidente de tráfico" y que tenían un código de causa externa distinto de accidente de tráfico, por entender que en estos casos había un error de codificación. Se incluyeron además entre los casos fallecidos por LT los códigos correspondientes a ciclistas V01 (.1.9), V10-11 (.3-.9), V17-18 (.3-.9) y V19 (.8.9), y los códigos de vehículo inespecífico V09.3 y V89.9.

La información sobre altas hospitalarias se obtuvo del CMBDAH de hospitales públicos y privados. La definición utilizada de alta hospitalaria por LT supone el cumplimiento de los siguientes criterios:

- Altas hospitalarias durante el periodo 2003-2005.

- Al menos un diagnóstico de lesión (Códigos CIE9-MC:800-959.9).

- Presencia de un código de causa externa de accidente de tráfico (códigos CIE9-MC: E810-819 o E826) o que constara como régimen de financiación "Aseguradora de accidente de tráfico" y no tuviera un código E distinto de accidente de tráfico.

- Ingreso urgente (en contraposición a ingreso programado).

La información sobre personas fallecidas se obtuvo del registro de mortalidad del Instituto de Estadística de la CM de los años 2003- 
2005. La definición utilizada de fallecido por AT incluyó los códigos de la CIE10: V01 $(.1,9)$, V02-V04 (.1,.9), V09 (.2,.3), V10-V11 (.3$.9)$, V12-V14(.3-.9), V17-18(.3-.9), V19(.4-.6) V19 (.8-.9), V20-28(.3-.9), V29-V79(.4-.9), V80 (.3-.5), V81-V82 (.1), V83-V86 (.0-.3), V87 (.0.8) o V89 (.2,.9).

La información sobre kilómetros recorridos y tiempo de desplazamiento se obtuvo de la Encuesta Domiciliaria de Movilidad de 2004 en la $\mathrm{CM}^{12}$, realizada por el Consorcio de Transportes de dicha Comunidad, última encuesta disponible de estas características. Se trata de un muestreo por conglomerados de viviendas y estratificado por zonas de transporte y tamaño del hogar. La encuesta, que incluyó a 34.942 familias y 84.317 personas mayores de 3 años, fue realizada mediante visita domiciliaria (alternativamente por teléfono, correo o internet) en días laborables durante los meses de octubre y noviembre de 2004. En ella se preguntaba por todos los viajes realizados por los miembros familiares en cualquier medio de transporte durante un día laborable, medio de transporte utilizado, tiempo invertido y dirección de origen y destino, calculándose luego la distancia recorrida mediante una matriz de orígenes-destinos.

Indicadores. Como indicadores epidemiológicos se utilizaron las tasas de altas hospitalarias y de mortalidad por 100.000 habitantes y las tasas de altas hospitalarias y de mortalidad por 1.000 millones de personas$\mathrm{km}$ recorridos y por un millón de personashoras de desplazamiento. En los tres indicadores el numerador recoge todas las LT. En los dos referidos a la exposición a la movilidad, el denominador se refiere sólo a vehículos privados. Para las tasas poblacionales se utilizó como denominador la suma de las poblaciones del padrón municipal de habitantes a 1 de enero de 2003, 2004 y 2005. En los indicadores referidos a la exposición a la movilidad se extrapoló a los tres años la movilidad estudiada en 2004.

Con el fin de disponer de casos suficientes para el análisis se decidió analizar el número de sujetos ingresados y sujetos fallecidos por LT correspondientes a los años 2003-05, próximos a la fecha de realización de la encuesta. Puesto que los registros de alta hospitalaria y de mortalidad no contemplan el día de la semana en que ocurrió la lesión, se extrapolaron los datos de la encuesta a todos los días del año, laborables y festivos. Para la población de 0 a 3 años, no contemplada en la encuesta, se extrapolaron los datos de distancia y tiempo de desplazamiento del grupo de edad de 4 a 15 años.

Análisis estadístico. A partir de los datos facilitados por el Consorcio de Transportes, se estimaron los kilómetros recorridos en vehículo privado y kilómetros por persona, las horas de desplazamiento utilizadas y la velocidad media en la CM durante los tres años de estudio, totales y desagregados por sexo y grupos de edad. Se calcularon las tasas de morbilidad y mortalidad mencionadas más arriba, totales y desagregadas por sexo y por los grupos de edad contemplados en la Encuesta de Movilidad.

Para los ingresos hospitalarios también se calculó el porcentaje de lesiones según su gravedad, de acuerdo con la escala Abbreviated Injury Scale (AIS), agrupando los valores máximos (MAIS) entre los estratos $\leq 2 \mathrm{y} \geq 3$, desagregados por sexo y edad. Los porcentajes se compararon mediante el test de $\chi^{2}$.

\section{RESULTADOS}

El número de kilómetros recorridos estimados por persona fue de 4.576,82 en hombres y 2.021,36 en mujeres. Los hombres recorrieron más kilómetros que las mujeres en todos los grupos de edad, especialmente, en el de 45-64 años. El grupo de edad que más kilómetros por persona recorrió fue el de 45-64 años en hombres y 25-64 en mujeres, mientras que los grupos de $0-15$ y 65 y más años fueron los menos viajeros. Los hombres, con una media de $25,3 \mathrm{~km} / \mathrm{h}$, viajaron a más velocidad que las mujeres en todos los grupos de edad, siendo la distribución de 


\begin{tabular}{|c|c|c|c|c|c|c|c|c|c|}
\hline \multicolumn{10}{|c|}{$\begin{array}{c}\text { Tabla } 1 \\
\text { Población de referencia en la Comunidad de Madrid en 2003-05, } \\
\text { horas de desplazamiento, kilómetros recorridos y velocidad, según sexo y edad }\end{array}$} \\
\hline & \multirow[t]{2}{*}{ Edad } & \multicolumn{2}{|c|}{$\begin{array}{c}\text { Población } \\
\text { de referencia }\end{array}$} & \multicolumn{2}{|c|}{$\begin{array}{c}\text { Horas } \\
\text { de desplazamiento } \\
\end{array}$} & \multicolumn{2}{|c|}{ Kilómetros recorridos } & \multirow{2}{*}{$\begin{array}{c}\text { Kilómetros / } \\
\text { persona }\end{array}$} & \multirow{2}{*}{$\begin{array}{c}\text { Velocidad } \\
\mathrm{Km} / \mathrm{h}\end{array}$} \\
\hline & & $\mathrm{n}$ & $\%$ & $\mathrm{n}$ & $\%$ & $\mathrm{n}$ & $\%$ & & \\
\hline \multirow{6}{*}{ Hombres } & $0-15$ & 1.384 .243 & 16,37 & 95.587 .790 & 6,26 & 1.388 .957 .586 & 3,59 & $1.003,41$ & 14,53 \\
\hline & $16-24$ & 1.025 .410 & 12,13 & 129.839 .381 & 8,50 & 3.174 .009 .765 & 8,20 & $3.095,36$ & 24,45 \\
\hline & $25-44$ & 3.132 .030 & 37,04 & 608.945 .172 & 39,85 & 16.715 .462 .967 & 43,19 & $5.336,94$ & 27,45 \\
\hline & $45-64$ & 1.901 .814 & 22,49 & 624.599 .083 & 40,88 & 15.292 .641 .833 & 39,52 & $8.041,08$ & 24,48 \\
\hline & $65+$ & 1.012 .258 & 11,97 & 57.249 .555 & 3,75 & 1.258 .107 .540 & 3,25 & $1.242,87$ & 21,98 \\
\hline & total & 8.455 .755 & 100,00 & 1.527 .907 .566 & 100,00 & 38.700 .450 .022 & 100,00 & $4.576,82$ & 25,33 \\
\hline \multirow{6}{*}{ Mujeres } & $0-15$ & 1.310 .740 & 14,51 & 96.834 .074 & 10,88 & 1.255 .862 .723 & 6,88 & 958,13 & 12,97 \\
\hline & $16-24$ & 997.598 & 11,04 & 68.979 .292 & 7,75 & 1.415 .677 .926 & 7,75 & $1.419,09$ & 20,52 \\
\hline & $25-44$ & 3.110 .595 & 34,44 & 372.198 .284 & 41,83 & 7.988.101.929 & 43,75 & $2.568,03$ & 21,46 \\
\hline & $45-64$ & 2.104 .041 & 23,29 & 263.661 .360 & 29,63 & 5.413 .406 .740 & 29,65 & $2.572,86$ & 20,53 \\
\hline & $65+$ & 1.509 .185 & 16,71 & 36.392 .473 & 4,09 & 734.098 .829 & 4,02 & 486,42 & 20,17 \\
\hline & total & 9.032 .159 & 100,00 & 889.846 .209 & 100,00 & 18.257 .247 .900 & 100,00 & $2.021,36$ & 20,52 \\
\hline \multirow{6}{*}{ Total } & $0-15$ & 2.694 .983 & 15,41 & 192.421 .864 & 7,97 & 2.644 .820 .309 & 4,66 & 981,39 & 13,74 \\
\hline & $16-24$ & 2.023 .008 & 11,57 & 198.818 .674 & 8,24 & 4.589 .687 .692 & 8,08 & $2.268,74$ & 23,08 \\
\hline & $25-44$ & 6.242 .625 & 35,70 & 981.143 .457 & 40,66 & 24.703 .564 .896 & 43,50 & $3.957,24$ & 25,18 \\
\hline & $45-64$ & 4.005 .855 & 22,91 & 888.260 .443 & 36,81 & 20.706 .048 .573 & 36,46 & $5.168,95$ & 23,31 \\
\hline & $65+$ & 2.521 .443 & 14,42 & 93.642 .028 & 3,88 & 1.992 .206 .369 & 3,51 & 790,11 & 21,27 \\
\hline & total $^{\mathrm{a}}$ & 17.487 .914 & 100,00 & 2.413 .298 .591 & 100,00 & 56.785 .967 .571 & 100,00 & $3.247,16$ & 23,53 \\
\hline
\end{tabular}

${ }^{a}$ El total de horas y kilómetros no coincide con la suma de parciales por ser estimaciones muestrales independientes.

\begin{tabular}{|c|c|c|c|c|c|c|c|c|c|}
\hline \multicolumn{10}{|c|}{$\begin{array}{c}\text { Tabla } 2 \\
\text { Personas hospitalizadas, fallecidas y gravedad de lesiones por tráfico según MAIS, } \\
\text { por sexo y edad. Comunidad de Madrid, 2003-05 }\end{array}$} \\
\hline & \multirow{3}{*}{ Edad } & \multirow{2}{*}{\multicolumn{2}{|c|}{$\begin{array}{c}\text { Personas } \\
\text { hospitalizadas }\end{array}$}} & \multicolumn{4}{|c|}{ MAIS $^{\text {a }}$} & \multirow{2}{*}{\multicolumn{2}{|c|}{$\begin{array}{l}\text { Personas } \\
\text { fallecidas }\end{array}$}} \\
\hline & & & & \multicolumn{2}{|c|}{$\leq 2$} & \multicolumn{2}{|c|}{$\geq 3$} & & \\
\hline & & $\mathrm{n}$ & $\%$ & $\mathrm{n}$ & $\%$ & $\mathrm{n}$ & $\%$ & $\mathrm{n}$ & $\%$ \\
\hline \multirow{6}{*}{ Hombres } & $0-15$ & 598 & 11,27 & 431 & 13,67 & 145 & 7,00 & 20 & 2,57 \\
\hline & $16-24$ & 1.235 & 23,27 & 710 & 22,52 & 513 & 24,77 & 123 & 15,81 \\
\hline & $25-44$ & 2.161 & 40,72 & 1.306 & 41,42 & 826 & 39,88 & 340 & 43,70 \\
\hline & $45-64$ & 880 & 16,58 & 494 & 15,67 & 374 & 18,06 & 186 & 23,91 \\
\hline & $65+$ & 433 & 8,16 & 212 & 6,72 & 213 & 10,28 & 109 & 14,01 \\
\hline & total & 5.307 & 100,00 & 3.153 & 100,00 & 2.071 & 100,00 & 778 & 100,00 \\
\hline \multirow{6}{*}{ Mujeres } & $0-15$ & 295 & 13,62 & 232 & 17,72 & 54 & 6,59 & 16 & 5,97 \\
\hline & $16-24$ & 311 & 14,36 & 167 & 12,76 & 137 & 16,73 & 44 & 16,42 \\
\hline & $25-44$ & 627 & 28,95 & 395 & 30,18 & 224 & 27,35 & 72 & 26,87 \\
\hline & $45-64$ & 433 & 19,99 & 263 & 20,09 & 168 & 20,51 & 65 & 24,25 \\
\hline & $65+$ & 500 & 23,08 & 252 & 19,25 & 236 & 28,82 & 71 & 26,49 \\
\hline & total & 2.166 & 100,00 & 1.309 & 100,00 & 819 & 100,00 & 268 & 100,00 \\
\hline
\end{tabular}


Figura 1

Tasas de morbilidad hospitalaria por lesión por tráfico, por sexo y edad. Comunidad de Madrid, 2003-05
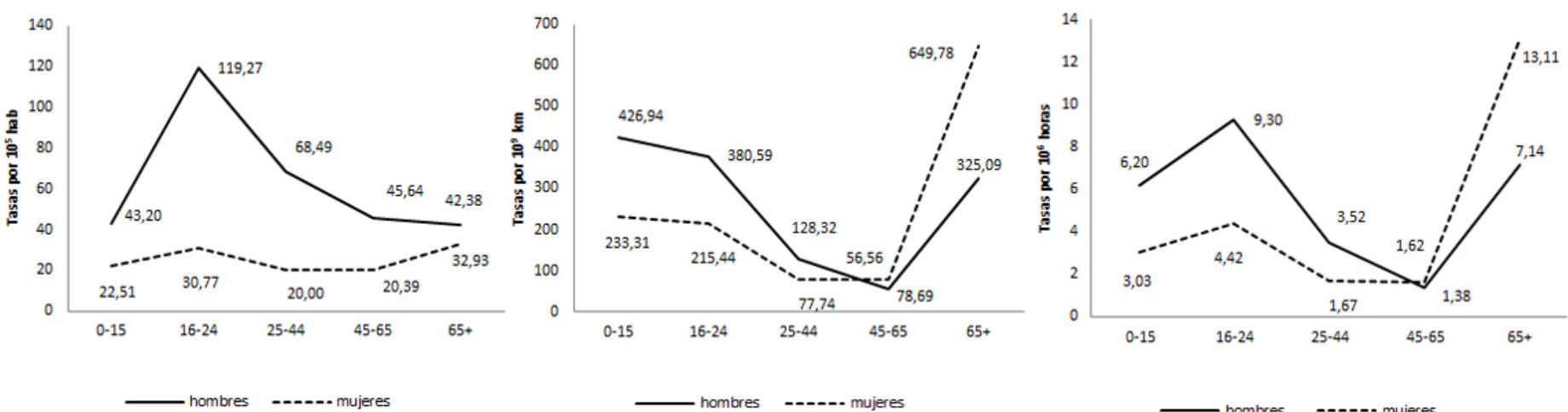

\section{Figura 2}

Tasas de mortalidad por lesión por tráfico, por sexo y edad. Comunidad de Madrid, 2003-05
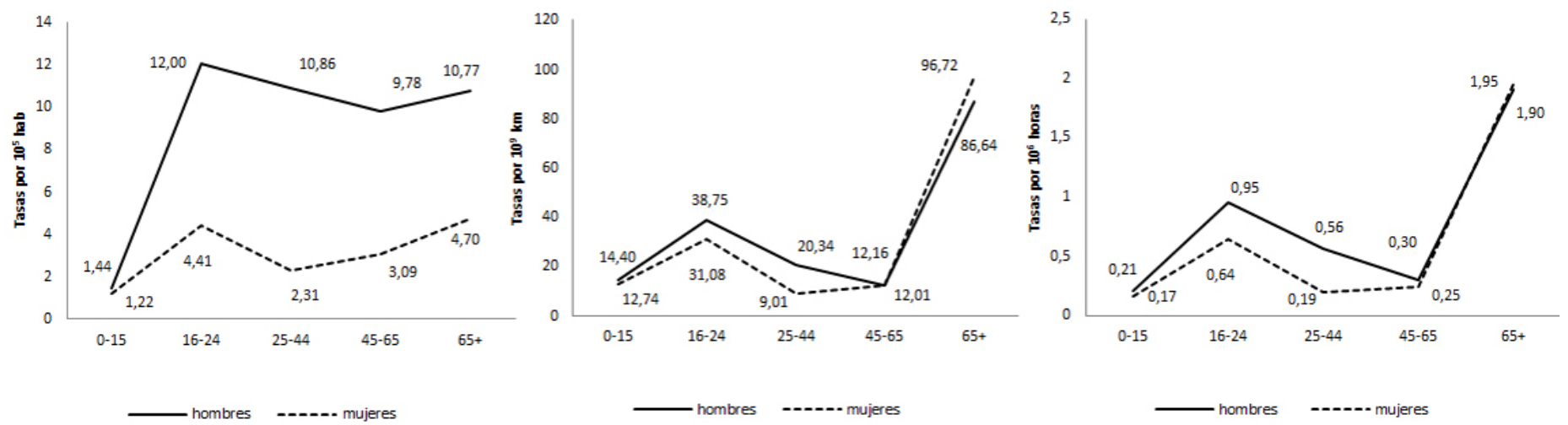
la velocidad por edades semejante en ambos sexos y el grupo de edad más veloz el de 25-44 años (tabla 1).

El número total de altas hospitalarias de lesión por AT registradas en el periodo 20032005 fue 7.473, de las cuales 5.307 fueron en hombres y 2.166 en mujeres. El número de personas fallecidas por AT en el mismo periodo fue de 1.046: 778 hombres y 268 mujeres. La edad media de las mujeres hospitalizadas (41,86 $\pm 23,54$ años) fue superior a la de los hombres $(34,15 \pm 18,20$ años) $(p<0,01)$. El mayor porcentaje de altas hospitalarias y fallecimientos en hombres se observó en el grupo de edad de $25-44$ años (40,72\% y 43,70\% respectivamente) y en las mujeres en los grupos de edad de $25-44(28,95 \%$ y $26,87 \%)$ y 65 y más años $(23,08 \%$ y $26,49 \%)$, siendo el porcentaje en este último grupo de edad casi el triple que el de hombres para hospitalizados $(8,16 \%)$ y casi el doble para fallecidos $(14,01 \%)$ (tabla 2).

La gravedad de las lesiones fue semejante para ambos sexos $(39,64 \%$ en hombres hospitalizados con valor MAIS $\geq 3$ y $38,49 \%$ de mujeres; $p=0,36)$, pero diferente por grupos de edad $(\mathrm{p}<0,01)$, siendo mayor en el grupo de edad de 65 y más años (49,18\% de hospitalizados con valor MAIS $\geq 3$ ) y menor en el grupo de 0 -15 años $(23,09 \%$ de hospitalizados con valor MAIS $\geq 3$ ) (tabla 2).

Las tasas totales de morbilidad y mortalidad por LT fueron superiores en hombres para los tres indicadores, especialmente para las tasas poblacionales. Por grupos de edad la morbilidad en hombres fue superior en los jóvenes de 16-24 años, para la tasa poblacional $(119,27$ casos por 100.000 habitantes) y la tasa de exposición temporal $\left(9,30\right.$ casos por $10^{6}$ horas $) y$ en el grupo de 0-15 años para la tasa de exposición espacial $\left(426,94\right.$ por $\left.10^{9} \mathrm{~km}\right)$. En mujeres la morbilidad fue mayor en el grupo de $65 \mathrm{y}$ más años: 32,93 casos por 100.000 habitantes, 649,78 casos por $10^{9} \mathrm{~km}$ y 13,11 casos por $10^{6}$ horas $(5,6$ y 5,5 veces superior que la media al considerar la tasa por kilómetros y horas respectivamente) (figura 1).
En cuanto a la mortalidad, el grupo de 65 años y más fue el más afectado en ambos sexos al considerar los indicadores temporoespaciales $(86,64$ hombres y 96,72 mujeres fallecidos por $10^{9} \mathrm{~km}$, y 1,90 hombres y 1,95 mujeres fallecidos por $10^{6}$ horas), mientras que la tasa poblacional mostró en hombres valores ligeramente superiores en el grupo 16-24 años (12,00 fallecidos por 100.000 habitantes) y en mujeres en el de 65 años y más (4,7 fallecidas por 100.000 habitantes). Cabe destacar aquí también el valor elevado de ambas tasas (por kilómetros y horas) para el grupo de mujeres de 65 y más años, 6,6 y 6,5 veces superior al valor medio de mujeres, respectivamente, pero, a diferencia de la morbilidad, que mostraba valores inferiores en hombres para este grupo de edad, la mortalidad en hombres de 65 y más años para un mismo tiempo de exposición o distancia recorrida se aproximó a la de mujeres de la misma edad (figura 2).

\section{DISCUSIÓN}

Los resultados de este estudio muestran que la morbi-mortalidad por lesiones graves de tráfico es superior en hombres en los tres indicadores estudiados. Las tasas poblacionales muestran valores superiores en el grupo de 16-24 años, sin embargo las tasas referidas a la exposición temporal y espacial reducen la predominancia masculina y juvenil y muestran tasas superiores en el grupo de 65 y más años para morbilidad en mujeres y para mortalidad en ambos sexos. Las tasas poblacionales, al no contemplar la exposición al riesgo, son superiores en los grupos de población que más utilizan el vehículo privado para su desplazamiento: los hombres y los grupos de edades medias.

La comparación de indicadores de morbimortalidad por LT llevada a cabo en este estudio constituye una novedad en nuestro país. En una investigación semilar, SantamariñaRubio y col. ${ }^{6}$ emplearon la medida personashoras para estimar el riesgo y las tasas poblacionales, por vehículos y por vehículos-km como medidas de comparación. 
Los resultados sobre distancias recorridas y velocidad se corresponden con lo esperado, de acuerdo con otros estudios publicados referidos sólo a conductores ${ }^{1,13,14}$. No obstante, en ellos se observa, sobre todo en hombres, que el mayor kilometraje anual y la máxima velocidad se dan a edades más jóvenes (en torno a 25-50 años para el kilometraje, frente a 45-64 años en nuestro caso, y 16-24 años para la velocidad, frente a 25-44 años en nuestro estudio). Llama la atención además la baja velocidad en el grupo 0-15 años, que podría atribuirse a la precaución de los conductores adultos que viajan con niños.

En los años estudiados España se situaba, por sus cifras de mortalidad, entre los países menos seguros de Europa ${ }^{15}$. Los resultados obtenidos de morbi-mortalidad por edad y sexo coinciden en general con los de otros estudios: menos lesiones en las edades medias de la vida y mayor incidencia en hombres, sobre todo en la mortalidad ${ }^{5,14,16-19}$. Este patrón por sexo y edad se mantiene independientemente de los diferentes indicadores manejados, tanto en cuanto al numerador (sólo conductores u ocupantes y peatones, hospitalizados o todos los lesionados) como al denominador (tasas poblacionales o diferentes medidas de exposición: personas-viajes, vehículos-km, personas-km o personas-horas). Solo en el caso de la morbilidad en conductores, la diferencia entre sexos se diluye o presentan tasas superiores las mujeres ${ }^{2,13,20}$, tal vez debido al menor kilometraje anual realizado por estas ${ }^{19}$.

La curva de edades (considerada a partir de 16 años) muestra normalmente en los distintos estudios en países industrializados la típica forma de $\mathrm{U}^{21}$. En el caso de lesiones no mortales, la curva suele mostrar mayor incidencia en jóvenes y tras un descenso en edades medias, un aumento en las edades mayores, de superior magnitud en el caso de lesiones graves, sin sobrepasar normalmente la tasa en jóvenes ${ }^{5,13,14,16-18,22-24}$. En nuestro caso, sin embargo, llama la atención el alto valor de las tasas de morbilidad referidas a exposición en mujeres de 65 y más años de edad, muy supe- rior al de las jóvenes ${ }^{5}$. Ello podría atribuirse en parte al peso de las peatonas hospitalizadas en este colectivo ${ }^{25}$, superior a otros grupos de edad y más determinante que en hombres, al presentar este grupo de mujeres valores bajos de exposición para los indicadores utilizados.

La curva de mortalidad por edades suele presentar también forma de U pero con tasas mayores en las edades más altas, superiores aunque no siempre a las de jóvenes de 16-24 años ${ }^{13,14,17,18,24,26}$. La elevación de las tasas de morbi-mortalidad en personas mayores parece explicarse sobre todo por su mayor fragilidad $^{18,21,22-24,26} \mathrm{y}$, en menor medida, por su mayor riesgo de colisión de tráfico, derivado de la realización de menos kilometraje anual, el denominado sesgo del kilometraje bajo ${ }^{21,24}$, 27-29. En nuestro caso, la presencia en ambos sexos de tasas de mortalidad referidas a exposición muy elevadas en el grupo de 65 y más años concuerda con lo observado en la morbilidad, si bien ahora las tasas son similares en ambos sexos: por cada 4 hombres hospitalizados de este grupo de edad hay uno fallecido, frente a una mujer fallecida por cada 7 hospitalizadas. La razón de esta discrepancia entre morbilidad y mortalidad por sexo en este grupo de edad puede ser una mayor letalidad para los hombres involucrados en colisiones de tráfico en comparación con la de las mujeres ${ }^{26}$, si bien un análisis por separado de las distintas formas de desplazamiento podría arrojar luz suplementaria sobre ello. En cualquier caso, a la luz de estos resultados, conviene llamar la atención sobre el elevado riesgo de LT en nuestro medio en la población de más edad.

Las tasas poblacionales de morbi-mortalidad son indicadores relevantes ampliamente utilizados en el estudio de las LT $^{30}$. Como todos los indicadores agregados, son útiles por su disponibilidad, pero no permiten comparar entre grupos o situaciones específicas ${ }^{4,31}$. En nuestro estudio observamos cómo las tasas poblacionales, frente a los indicadores que contemplan la exposición al riesgo, minimizan la morbi-mortalidad en las edades extre- 
mas y en mujeres en general, situaciones asociadas a la realización de menor kilometraje anual y por tanto menor exposición (menor uso del vehículo), y ocurre lo contrario en las edades medias y hombres, situaciones asociadas a la realización de mayor kilometraje anual (mayor uso del vehículo).

Para la puesta en marcha de políticas de seguridad del transporte, son necesarias medidas de exposición ${ }^{32}$. Aun cuando el concepto de exposición en este ámbito y su medida no están plenamente establecidos ${ }^{33}$, los indicadores de exposición que relacionan los casos con las personas-distancia recorrida se hallan entre los más utilizados ${ }^{1,3,30}$, y los que utilizan personas-horas en tránsito son incluso preferidos por algunos autores ${ }^{2,16}$.

Los indicadores basados en la distancia realzan la morbi-mortalidad en las zonas densas, de tráfico lento ${ }^{16} \mathrm{y}$ adolecen del denominado sesgo de kilometraje bajo ${ }^{4,31}$. Los indicadores temporales por su parte, como se observa en nuestro estudio, resaltan la mayor morbimortalidad en hombres, probablemente debido a que conducen más rápido ${ }^{1}$. La relación entre tiempo, espacio y riesgo es compleja $^{1,2}$. La velocidad juega un papel importante en esta relación, por ello ha de ser tenida en cuenta y algunos autores proponen que la exposición sea medida combinando de algún modo el espacio recorrido y el tiempo utilizado $^{1}$. Pero además, no existe una relación lineal entre exposición y efecto en las LT, pues dicha relación se halla mediada por factores ambientales y de comportamiento individual $^{27,30}$. Por ello, a falta de criterios generales definitivos, la elección del indicador ha de realizarse de acuerdo con el uso que se le quiera dar ${ }^{31}$. En cualquier caso, a diferencia de otros autores ${ }^{32}$, a nuestro juicio el tiempo utilizado parece adaptarse mejor a la diversidad de situaciones que se ofrecen en las comparaciones: diferentes conductores y vehículos, diferentes carreteras, condiciones de tráfico... La velocidad suele adaptarse a tales variaciones y aconsejaría por tanto el uso de un indicador temporal ${ }^{2}$.
Existen ciertas limitaciones metodológicas en este estudio, consecuencia de las limitaciones propias del $\mathrm{CMBDAH}^{10}$. Este registro no discrimina ingresos y reingresos y adolece de falta de exhaustividad de la codificación $\mathrm{E}$ (72,7\% de cobertura de cumplimentación del código de causa externa en 2003-2005 entre las altas hospitalarias por lesión). Esto último implica seguramente una infraestimación de las tasas de morbilidad. Tampoco permite conocer la fecha de origen de la LT, lo que ha obligado a extrapolar los datos de la Encuesta Domiciliaria de Movilidad, realizada en días laborables, a los días festivos. Teniendo en cuenta que, según los datos de la Encuesta de Movilidad de las Personas Residentes en España MOVILIA 2006/200734, el número estimado de desplazamientos en coche o moto, en la Comunidad de Madrid, para esos años, fue similar en día laborable y fin de semana, puede suponerse que dicha extrapolación no afectará de forma importante a la representatividad diaria de la muestra. Sin embargo, según la misma fuente, el número de desplazamientos en coche o moto en España es algo superior en fin de semana en las edades extremas (014 años $y \geq 65$ años) e inferior en el resto de edades, lo que supondría una cierta sobrestimación de las tasas en las edades extremas e infraestimación en el resto. Finalmente, dado el alto porcentaje de casos con códigos inespecíficos en el CMBDAH $(48,6 \%$ de casos de LT con código E desconocido o correspondiente a persona y tipo de accidente de tránsito desconocido: código E 819.9), es imposible discriminar entre LT en vehículo público y privado y entre peatones y no peatones, por lo que todas las LT han sido forzosamente contabilizadas en el numerador de los indicadores que recogen la exposición, mientras que los denominadores de éstos se refieren sólo a kilómetros recorridos o tiempo transcurrido en vehículos privados. Aunque parecería metodológicamente más correcto incluir también en los denominadores los kilómetros u horas empleados en el desplazamiento en vehículos públicos (autobús, tren y metro) y en el desplazamiento de peatones, hemos preferido no hacerlo, dado que ello enmascararía el riesgo 
de los ocupantes de vehículos privados, los más usualmente accidentados. Puede suponerse fácilmente que en la inmensa mayoría de hospitalizados, ocupantes de un vehículo que sufre una colisión, el vehículo sea de uso privado. Y, por otro lado, el riesgo de ser atropellados depende también en gran medida de la cantidad de tráfico rodante, mientras que el número de lesionados ocupantes de un vehículo privado no parece tener a priori relación alguna con la movilidad de los peatones, de modo que el indicador resultante tendría una significación confusa si incluyéramos dicha movilidad en el denominador. A pesar de tales limitaciones, la información sobre morbilidad contenida en el CMBDAH es, además de fácilmente asequible, insustituible por otras fuentes.

Otra limitación de este estudio proviene de la extrapolación realizada de los datos de movilidad de 4-15 años al grupo 0-3 años. Aun cuando podrían haberse suprimido del estudio los casos de 0-3 años, hemos preferido no hacerlo con el fin de trabajar con indicadores relativos a grupos de edad tradicionales, aptos también para la comparación y vigilancia epidemiológica. Pensamos que la magnitud del sesgo posiblemente introducido al hacerlo no desvirtúa en absoluto las conclusiones obtenidas en el estudio.

En conclusión, los dos indicadores basados en la exposición a la movilidad reducen la preponderancia masculina y juvenil de las tasas de morbi-mortalidad observada en el indicador poblacional y elevan las tasas en personas mayores, que llegan a ser predominantes para la morbilidad en mujeres y sobre todo para la mortalidad en ambos sexos. Son aquellos, por tanto, indicadores más precisos en la estimación del riesgo de padecer una LT y permiten que afloren hipotéticos factores de riesgo, como la fragilidad en las personas mayores o la menor pericia en la conducción de quienes menos kilometraje realizan. Los resultados así obtenidos ponen de manifiesto el elevado riesgo de LT en nuestro medio en la población de más edad. Aunque la utilización de un tipo de indicador determinado depende de los objetivos de análisis y los dos indicadores referidos a la exposición a la movilidad muestran resultados semejantes por edad y sexo, la mayor flexibilidad del indicador temporal en relación con las diferentes circunstancias de riesgo ambientales y personales aconsejaría utilizar más este indicador que el indicador espacial.

\section{AGRADECIMIENTOS}

Al Consorcio Regional de Transportes de Madrid, en especial a Maite Antón Millán, por poner a nuestra disposición los datos requeridos de la Encuesta Domiciliaria de Movilidad de 2004 en la Comunidad de Madrid.

\section{BIBLIOGRAFÍA}

1. Chipman ML, MacGregor CG, Smiley LA, Lee-Gosselin M. Time vs. distance as measures of exposure in driving surveys. Accid Anal Prev. 1992; 24:679-84.

2. Chipman ML, MacGregor CG, Smiley LA, Lee-Gosselin M. The role of exposure in comparisons of crash risk among different drivers and driving environments. Accid Anal Prev. 1993; 25:207-11.

3. SafetyNet. Deliberable 2.1. State of the art report on risk and exposure data. National Technical University of Athenas [edición electrónica]. 2005 [citado el 12/2/2015]. Disponible en http://erso.swov.nl/safetynet/ fixed/WP2/Deliverable\%20wp $\% 202.1 \% 20$ state $\% 20$ of $\% 20$ the $\% 20$ art.pdf

4. Wundersitz LN, Hutchinson TP. Identifying and improving exposure measures. Centre for Automotive Safety Research. University of Adelaide [edición electrónica]. 2008 [citado el 12/2/2015]. Disponible en http://digital. library.adelaide.edu.au/dspace/handle/2440/50537

5. Santamariña-Rubio E, Pérez K, Olabarria M, Novoa AM. Gender differences in road traffic injury rate using time travelled as a measure of exposure. Accid Anal Prev. 2014; 65:1-7.

6. Santamariña-Rubio E, Pérez K, Olabarria M, Novoa AM. Measures of exposure to road traffic injury risk. Inj Prev. 2013; 19:436-9.

7. Broughton J, Keigan M, Yannis G, Evgenikos P, Chaziris A, Papadimitriou E et al. Estimation of the real number of road casualties in Europe. Saf Sci. 2010; 48:36571 . 
8. Pérez C, Cirera E, Borrell C, Plasència A. Motor vehicle crash fatalities at 30 days in Spain. Gac Sanit. 2006; 20:108-15.

9. Andreassen D. Population and registered vehicle data vs road deaths. Accid Anal Prev 1991; 23: $343-$ 51.

10. Pérez C, Cirera E, Plasència A. Estudio de la mortalidad a 30 días por accidente de tráfico (EMAT-30). Madrid: Ministerio de Sanidad y Consumo; 2004. [citado el 12/2/2015]. Disponible en http://www.msssi. gob.es/ciudadanos/accidentes/docs/morAccTrafico. pdf

11. Grupo de trabajo de la Sociedad Española de Epidemiología sobre la medida del impacto en la salud de las lesiones por accidente de tráfico en España. Indicadores de morbilidad y mortalidad de lesión por accidente de tráfico. Madrid: Ministerio de Sanidad y Consumo. Información y Estadísticas Sanitarias; 2007.

12. Encuesta Domiciliaria de Movilidad de 2004 en la Comunidad de Madrid. Documento de síntesis [internet]. Madrid: Consorcio de Transportes de Madrid;2005. [citado el 27/5/2014]. Disponible en: http:// prueba.crtm.es/media/157705/edm_2004.pdf.

13. Ryan GA, Legge M, Rosman D. Age related changes in drivers' crash risk and crash type. Accid Anal Prev. 1998; 30:379-87.

14. Beck LF, Dellinger AM, O’Neil ME. Motor vehicle crash injury rates by mode of travel, United States: using exposure-based methods to quantify differences. Am J Epidemiol. 2007; 166:212-8.

15. Twisk DAM, Stacey C. Trends in young driver risk and countermeasures in European countries. J Safety Res. 2007; 38:245-57.

16. Blaizot S, Papon F, Haddak MM, Amoros E. Injury incidence rates of cyclists compared to pedestrians, car occupants and powered two-wheeler riders, using a medical registry and mobility data, Rhône County, France. Accid Anal Prev. 2013; 58: 35-45.

17. Massie DL, Campbell KL, Williams AF. Traffic accident involvement rates by driver age and gender. Accid Anal Prev. 1995; 27:73-87.

18. Evans L. Risks older drivers face themselves and threats they pose to other road users. Int J Epidemiol. 2000; 29: 315-22.

19. Massie DL, Green PE, Campbell KL. Crash involvement rates by driver gender and the role of average annual mileage. Accid Anal Prev. 1997; 29:675-85.
20. Lourens PF, Vissers JAMM, Jessurun M. Annual mileage, driving violations, and accident involvement in relation to drivers' sex, age, and level of education. Accid Anal Prev. 1999; 31:593-7.

21. Langford J, Koppel S. Epidemiology of older driver crashes - Identifying older driver risk factors and exposure patterns. Transp Res Part F. 2006; 9:309-21.

22. Clark DE, Wildner M, Bergmann KE. Injury mortality in East Germany. Am J Public Health. 2000; 90: 1761-4.

23. Meuleners LB, Harding A, Lee AH, Legge M. Fragility and crash over-representation among older drivers in Western Australia. Accid Anal Prev. 2006; 38:1006-10.

24. Mitchell CGB. The licensing and safety of older drivers in Britain. Accid Anal Prev. 2013; 50:73241.

25. Suelves JM, Salas T, Cabezas C. Hospitalització urgent per lesions no intencionades a Catalunya l'any 2010. Butll Epidemiol Catalunya. 2011; 32:143-53.

26. Li G, Braver ER, Chen LH. Fragility versus excessive crash involvement as determinants of high death rates per vehicle-mile of travel among older drivers. Accid Anal Prev. 2003; 35:227-35.

27. Janke MK. Accidents, mileage, and the exaggeration of risk. Accid Anal Prev 1991; 23:183-8.

28. Langford J, Methorst R, Hakamies-Blomqvist L. Older drivers do not have a high crash risk- A replication of low mileage bias. Accid Anal Prev. 2006; 38:574-8.

29. Hakamies-Blomqvist L, Raitanen T, O’Neill D. Driver ageing does not cause higher accident rates per km. Transp Res Part F. 2002; 5:271-4.

30. Farchi S, Molino N, Rossi PG, Borgia P, Krzyzanowski M, Dalbokova D et al. Defining a common set of indicators to monitor road accidents in the European Union. BMC Public Health. 2006; 6:183-94.

31. Bobevski I, Clark B, Lenné M, Keal M, Diamantopoulou K, Cameron M. Development of road safety behaviour, travel and exposure surveys in Victoria. Victoria: Monash University Accident Research Center;2007.

32. European Transport Safety Council. Exposure data for travel risk assessment: current practice and future needs in the EU. Brussels. 1999 
33. Hutchinson TP, Wundersitz LN, Anderson RWG, Kloeden CN. Exposure to risk on the roads. Sydney: Proceedings of 2009 Australasian Road Safety Research, Policing and Education Conference. Sidney: Road Traffic Authority of New South Wales; 2009. p 190-200.

34. Ministerio de Fomento. Encuesta de Movilidad de las personas residentes en España (MOVILIA 2006/2007). [citado el 16/4/2015]. Disponible en:http:// www.fomento.gob.es/MFOM/LANG_CASTELLANO/ ATENCION_CIUDADANO/INFORMACION_ESTADISTICA/Movilidad/Movilia2006_2007/ 